\title{
Charge Redistribution and Phonon Entropy of Vanadium Alloys
}

\author{
O. Delaire and B. Fultz \\ W. M. Keck Laboratory, California Institute of Technology, Mail 138-78, Pasadena, California 91125, USA
} (Received 11 January 2006; revised manuscript received 20 September 2006; published 11 December 2006)

\begin{abstract}
The effects of alloying on the lattice dynamics of vanadium were investigated using inelastic neutron scattering. Phonon densities of states were obtained for bcc solid solutions of $\mathrm{V}$ with $3 d, 4 d$, and $5 d$ transition metal solutes, from which vibrational entropies of alloying were obtained. A good correlation is found between the vibrational entropy of alloying and the electronegativity of transition metal solutes across the $3 d$ row and down columns of the periodic table. First-principles calculations on supercells matching the experimental compositions predicted a systematic charge redistribution in the nearestneighbor shell around the solute atoms, also following the Pauling and Watson electronegativity scales. The systematic stiffening of the phonons is interpreted in terms of the modified screening properties of the electron density around the solutes.
\end{abstract}

DOI: 10.1103/PhysRevLett.97.245701

Over the last decade, experimental and theoretical investigations have shown that changes in vibrational entropy play an important role in the thermodynamics of solid phases [1-3]. We recently showed that for alloys containing a few percent solute, the vibrational entropy of mixing can sometimes overcome the configurational entropy from disorder, leading to a total entropy of mixing with a negative sign [4]. A systematic understanding of how vibrational entropy affects alloying and compound formation has remained elusive, however. The present experiment was designed to identify trends in alloying transition metals with vanadium, an element that has some advantages for determining phonon spectra by inelastic neutron scattering [4]. In the present study we report results for solutes across the $3 d$ row and down three columns on the periodic table. This range of solutes allows a test of correlations between the vibrational entropy of alloying and the Hume-Rothery parameters of metallic radius, electronegativity, and electron-to-atom ratio, with atom mass added to this list. Similar considerations were used for compound formation [5], where solute mass and metallic radius gave reasonable correlations, and a "bond stiffness versus bond length" model [6] has also shown promise.

Here we report a smooth trend for bcc V alloyed with solutes across the $d$ series: solutes to the left of vanadium induce a softening of the phonon modes, whereas a systematic stiffening is found for elements to the right of V. In spite of substantial variations in solute mass, metallic radius, and electron-to-atom ratio, we find that the vibrational entropy of alloying correlates well with the Pauling electronegativity for all $3 d, 4 d$, and $5 d$ transition metal solutes. Parallel trends in charge transfer were found from first-principles calculations of the charge distribution. The electronic structure calculations identified a softening and stiffening trend of the first-nearest-neighbor $(1 \mathrm{NN})$ shell around the solutes, in good agreement with our phonon measurements.

Alloys of vanadium with 6.25 at. \% solutes were prepared by arc-melting under an ultrahigh purity (UHP)
PACS numbers: $64.75 .+\mathrm{g}, 61.12 .-\mathrm{q}, 63.20 . \mathrm{Mt}, 63.70 .+\mathrm{h}$

argon atmosphere. The ingots were subsequently coldrolled to thicknesses for scattering $10 \%$ of the incident neutrons (the brittle $\mathrm{V}-\mathrm{Zr}$ and $\mathrm{V}-\mathrm{Hf}$ samples were crushed to form granules of appropriate size). All samples were then annealed in UHP Ar at $1000{ }^{\circ} \mathrm{C}$ for $1 \mathrm{~h}$ and quenched into ice brine. X-ray diffractometry showed all samples to be bcc solid solutions, and the x-ray diffraction patterns were refined to obtain lattice parameters. Alloy densities were also measured, from which a second value of lattice parameter was derived. Details are given in a supplementary publication [7].

Time-of-flight inelastic neutron scattering spectra were measured at room temperature with the LRMECS chopper spectrometer at the IPNS spallation neutron source at the Argonne National Laboratory. The incident energy was $50 \mathrm{meV}$ and the wide angular range of detector coverage allowed for momentum transfers between 0.5 and $9 \AA^{-1}$ at zero energy transfer. The FWHM energy resolution was $1.0 \mathrm{meV}$ at $40 \mathrm{meV}$ positive energy transfer, increasing to $2.4 \mathrm{meV}$ at the elastic line. All spectra were normalized by the total incident flux and sample mass, and were corrected for detector efficiency, time-independent background, and scattering from the empty sample container. A standard procedure was used to remove effects of thermal phonon occupation and multiphonon scattering [8]. This analysis uses the incoherent-scattering approximation, which is appropriate for solid solutions of vanadium when solutes that scatter coherently are distributed randomly.

For pure elements of cubic crystals, this data reduction procedure provides the phonon density of states (DOS) [9]. For an alloy, however, the result is a "neutron-weighted" phonon DOS. Because different isotopes have different cross sections for phonon scattering, in proportion to the neutron cross section over mass $\sigma / M$, the modes with large projections onto elements of bigger $\sigma / M$ are emphasized over modes with large projections onto elements with smaller $\sigma / M$. The solutes $\mathrm{Ti}, \mathrm{Co}, \mathrm{Zr}$, and $\mathrm{Cr}$ could present only small problems with neutron weighting for any phonon distribution (see [7]). Specific assessments of neutron 
weighting for $\mathrm{Ni}, \mathrm{Pd}$, and $\mathrm{Pt}$ were performed in our previous work [4] by comparing vibrational entropies calculated from the neutron-weighted DOS and its deweighted counterpart. The effect is potentially large for $\mathrm{Pd}$, for which $\sigma / M$ differs significantly from that of $\mathrm{V}$, and $\mathrm{Pd}$ has a significantly different phonon partial DOS curve. Even for the case of Pd, there is only a small effect of neutron weighting on the vibrational entropy of alloying, changing it from $-0.17 \pm 0.01 k_{B} /$ at to $-0.15 \pm$ $0.01 k_{B} /$ at. This is comparable to the error from counting statistics in the measured spectra. Similar or smaller effects were found for $\mathrm{Ni}$ and $\mathrm{Pt}$ solutes. The dominant contributions to the vibrational entropy originate from $\mathrm{V}$ atoms themselves, as discussed further below.

Phonon DOS curves for $3 d$ solutes are shown in Fig. 1. For V-6.25\% Ti, the phonon DOS is softer than in pure V. For $\mathrm{Cr}, \mathrm{Fe}, \mathrm{Ni}$, and Co solutes the phonon DOS is stiffer than in pure $\mathrm{V}$, and this stiffening increases smoothly across the $3 d$ series. It appears that all phonon modes follow this stiffening trend. Phonon DOS curves for solutes down the Ti, V, and Ni columns are shown in Fig. 2. Down the column to the left of $\mathrm{V}$, the solutes $\mathrm{Ti}, \mathrm{Zr}$, and $\mathrm{Hf}$ induce an increased softening in the phonon DOS. The solutes $\mathrm{Nb}$ and $\mathrm{Ta}$, isoelectronic to $\mathrm{V}$, have very small effects on the $\mathrm{V}$ phonons. $\mathrm{Nb}$ induces a slight softening, but, surprisingly, heavy Ta solutes have no detectable effect on the $\mathrm{V}$ phonon DOS, consistent with the previous measurement of Syrykh et al. on $\mathrm{V}-5.7 \% \mathrm{Ta}$ [10]. On the other hand, the solutes $\mathrm{Ni}$, $\mathrm{Pd}$, and $\mathrm{Pt}$ induce a big stiffening of the phonon DOS, increasing systematically from Ni to Pd to Pt. In each case, the shift affects all phonon modes, although there is also some restructuring of the phonon DOS. Also, we have previously shown using a Born-von Kármán lattice dy-

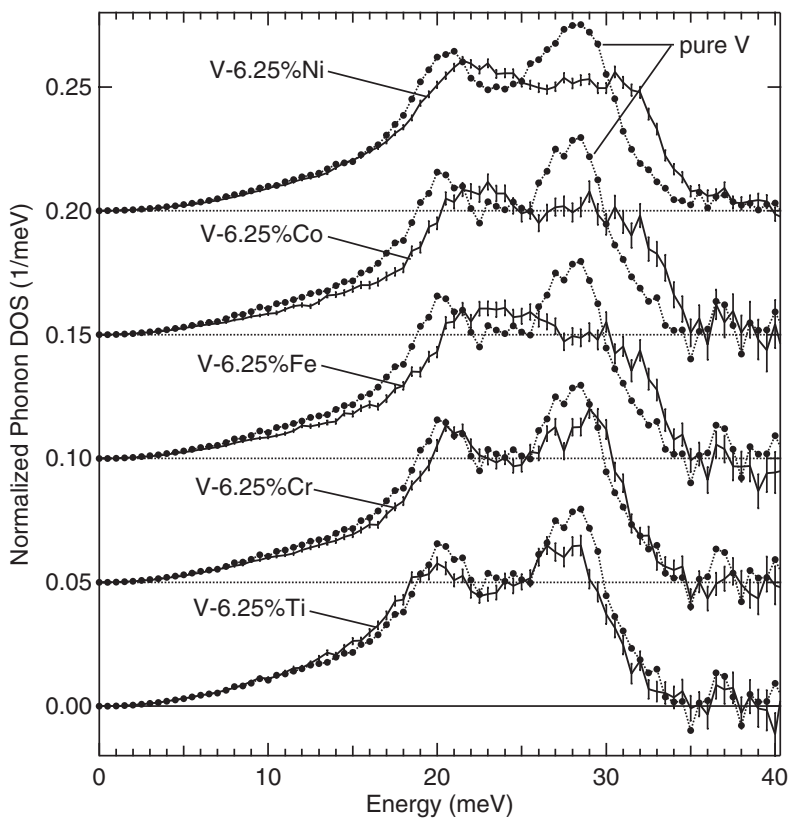

FIG. 1. Neutron-weighted phonon DOS for solutes across the $3 d$ series. Markers, pure V; solid lines, V-6.25\%X. namics model that $\mathrm{Pd}$ and $\mathrm{Pt}$ solutes exhibit resonance modes around $12 \mathrm{meV}$. However, these modes affect only a small portion of the total DOS for solute concentrations of $6.25 \%$.

In the quasiharmonic approximation, the vibrational entropy, $S_{\mathrm{vib}}$, is

$$
S_{\text {vib }}=3 k_{B} \int_{0}^{E_{\max }}[(n+1) \ln (n+1)-n \ln (n)] g(E) d E,
$$

where $n$ is the Bose-Einstein distribution and $g(E)$ is the DOS. We define the vibrational entropy of alloying as $\Delta S_{\text {vib }}^{\mathrm{al}}=S_{\mathrm{vib}}^{\mathrm{V}-\mathrm{X}}-S_{\mathrm{vib}}^{\mathrm{V}}$. The results are tabulated in [7] and plotted in Fig. 3. The vibrational entropies of alloying are large for solutes down the Ti column and for solutes at the end of the transition metal series (comparable, in fact, to the configurational entropy of mixing), whereas $\mathrm{Cr}$ and solutes isoelectronic with $\mathrm{V}$ (i.e., $\mathrm{Nb}, \mathrm{Ta}$ ) have small effects on $\Delta S_{\text {vib }}^{\text {al }}$.

Hume-Rothery parameters that can underlie changes in bond stiffness are the size of the solute atom, and its electronic properties, i.e., the number of $d$ electrons and electronegativity. A volume correlation presents an incomplete picture - for solutes across the $3 d$ series the smaller volumes correlate to stiffening, whereas down the $\mathrm{Ni}$ column from $\mathrm{Ni}$ to $\mathrm{Pt}$ the larger volumes correlate to stiffening. (From Ni to Pt the strongest stiffening occurs when there is, somewhat surprisingly, only a slight change of lattice parameter [7].) The vibrational entropy of alloying follows approximately the number of $d$ electrons of the solute, $N_{d}$, as shown in Fig. 3. There are a number of discrepancies, however, especially down the $\mathrm{Ni}$ and $\mathrm{Ti}$ columns of the periodic table where $N_{d}$ is constant. The systematic stiffening of the phonon DOS for solutes across the $3 d$ series and down the Ni column is opposite to the effect predicted from the increasing solute mass. The experimental results therefore show that the trends in vibrational entropy of alloying originate from changes in the interatomic force constants owing to the alteration of elec-

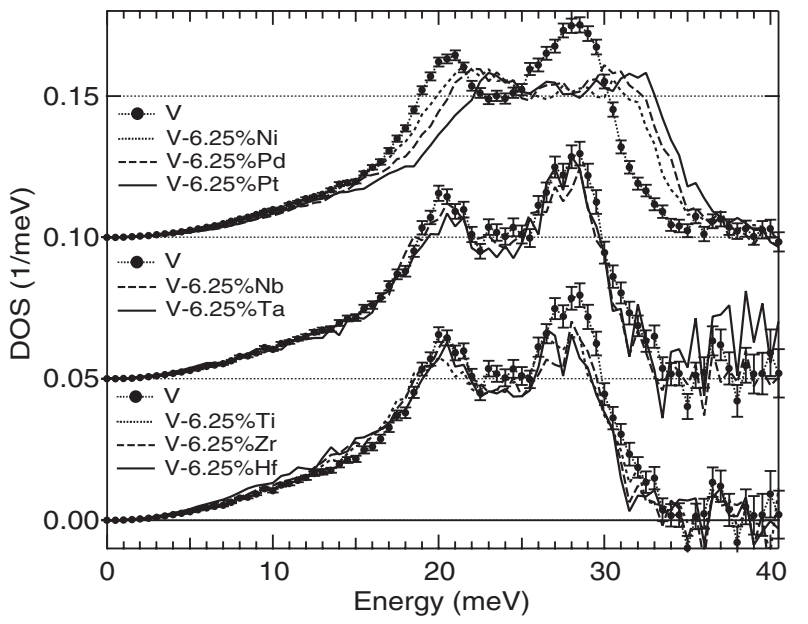

FIG. 2. Neutron-weighted phonon DOS for solutes down the $\mathrm{Ni}, \mathrm{V}$, and Ti columns. 


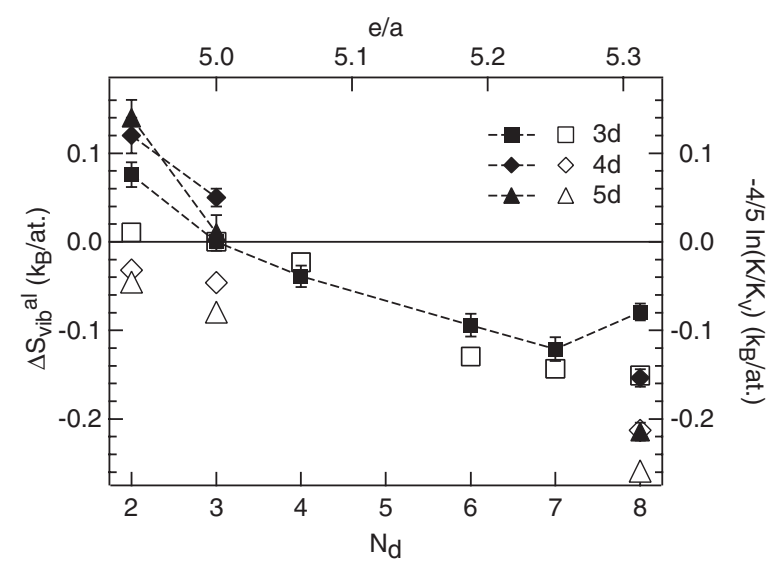

FIG. 3. Neutron-weighted vibrational entropy of alloying (solid symbols) and entropy change from calculated breathing mode stiffness (open symbols), plotted versus formal number of $d$ electrons of the solute ( 3 for $\mathrm{V}$ ) and electron-to-atom ratio for the alloy, $e / a$.

tronic structure around the solute atoms. The solute concentrations are relatively dilute, so it is difficult for the partial DOS of the solute atoms to account for the large effects observed. (Small contributions from changes in the solute partial phonon DOS are also consistent with the small effects of neutron weighting on the vibrational entropy.) Each solute is surrounded by $8 \mathrm{~V}$ first-nearest neighbors and $6 \mathrm{~V}$ second neighbors, however, which directly sense the solute atom. The experimental results suggest that it is not directly the vibrational modes projected onto the solute atoms that are responsible for the observed trends, but rather the influence of the solutes on the bonds to their vanadium neighbors.

The V-X alloys were further investigated by density functional theory calculations. Supercells containing 16 atoms $(2 \times 2 \times 2$ cubic cells), with the solute atom at the central site, were used to model the disordered V-6.25\%X alloys. The calculations were performed with both WIEN2K (linearized augmented plane waves) [11] and VASP (projector augmented waves) [12,13]. The WIEN2K simulations used the Perdew-Burke-Ernzerhof generalized gradient approximation (GGA) [14], while VASP calculations were conducted with the Perdew-Wang GGA [15]. The $2 \times 2 \times$ 2 supercell calculations were conducted with a $10 \times 10 \times$ 10 Monckhorst-Pack [16] k-point grid in WIEN2K and a $16 \times 16 \times 16$ grid in VASP, corresponding to 35 and $120 \mathbf{k}$ points in the irreducible portion of the Brillouin zone, respectively. The WIEN2K calculations were performed with $R_{\mathrm{MT}} \times k_{\max }=7.0$, while VASP calculations used the "accurate" setting for the kinetic energy cutoff. For the magnetic solutes $\mathrm{Fe}, \mathrm{Co}$, and $\mathrm{Ni}$, spin-polarized calculations were performed on the relaxed $2 \times 2 \times 2$ supercells, indicating no magnetization on the solute atom, so most simulations were performed without spin polarization.

The total energy was minimized with respect to the supercell volume and the atom positions. The relaxation results from WIEN2K and VASP were in excellent agreement and the calculated change in lattice parameter upon alloying was within the error bars of the experimental measurements (see [7]), although the absolute lattice parameter was consistently underestimated by about $1 \%$. Geometry optimizations were also conducted on $3 \times 3 \times 3$ supercells with composition $\mathrm{V}_{53} \mathrm{X}_{1}$, and these indicated that most of the relaxation occurs in the $1 \mathrm{NN}$ shell around $\mathrm{X}$, with the exception of the V-Ni system, where the $2 \mathrm{NN}$ bond around $\mathrm{Ni}$ solutes contracts more than the $1 \mathrm{NN}$ bond. The changes in $1 \mathrm{NN}$ bond length calculated with $2 \times 2 \times 2$ and $3 \times$ $3 \times 3$ supercells were in good agreement, indicating that effects are localized around each solute.

The deformation energies for breathing modes of $1 \mathrm{NN} \mathrm{V}$ atoms around the different solute atoms (motions along $\langle 111\rangle$ ) were calculated (VASP, $2 \times 2 \times 2$ supercells) as a functions of displacement amplitude. The potentials were largely harmonic, with harmonic stiffnesses obtained from fits to 5th-order polynomials (see [7]). Assuming the partial DOS for these $1 \mathrm{NN} \mathrm{V}$ solute atoms scales as $\left(K / K_{\mathrm{V}}\right)^{1 / 2}$, a 6.25 at. $\%$ solute concentration would change $\Delta S_{\text {vib }}^{\text {al }}$ by $-4 / 5 k_{B} \ln \left(K / K_{\mathrm{V}}\right)$, with $K$ the stiffness for the $1 \mathrm{NN} \mathrm{V}$ atoms and $K_{\mathrm{V}}$ the stiffness for the other $\mathrm{V}$ atoms (the solute partial DOS is ignored). This estimate of $\Delta S_{\text {vib }}^{\mathrm{al}}$ is plotted on the right scale of Fig. 3. The agreement with the experimental results is generally good in both trend and magnitude.

Calculations of atomic charges were performed using the topological analysis of the electron density introduced by Bader [17,18]. A systematic charge transfer was found between the Bader volumes for the solutes and their 1NN atoms, with group IV elements losing electrons to the host vanadium (which is in group $\mathrm{V}$ ) and elements to the right of group V gaining electrons [7]. The $2 \mathrm{NN}$ and $3 \mathrm{NN}$ shells appear not to play important roles in this charge transfer. The calculated charge transfers are larger than generally assumed in metallic alloys, but it is known that the Bader scheme tends to predict larger values than other standard methods, although the trends are usually in agreement [19]. The trend obtained for the charge transfer seems natural in the view of the solute electronegativity, and a particularly good linear correlation is observed with the electronegativity scale of Watson and Bennett [20].

Figure 4 shows the correlation of $\Delta S_{\text {vib }}^{\text {al }}$ with difference in Pauling's electronegativity between host and solute, $\Delta \chi$, and the calculated charge transfer to the solute, $\Delta Q_{\mathrm{X}}$. This graph shows that $\Delta S_{\text {vib }}^{\text {al }}$ varies linearly with the electronegativity of the solute, even for the large and heavy solutes of the $4 d$ and $5 d$ rows. The correlation with electronegativity for our $6.25 \%$ solute concentrations, weighted with experimental error bars, is $\Delta S_{\text {vib }}^{\text {al }} / k_{B}=$ $-0.34( \pm 0.06) \Delta \chi$. This correlation is successful both across the $3 d$ row and down three columns of the periodic table. We also find a good correlation with the charge transfer computed from first-principles methods: $\Delta S_{\mathrm{vib}}^{\mathrm{al}} / k_{B}=-0.11( \pm 0.01) \Delta Q_{\mathrm{X}} / e$. These results indicate that a change in the bonding between solute and $1 \mathrm{NN} \mathrm{V}$ 


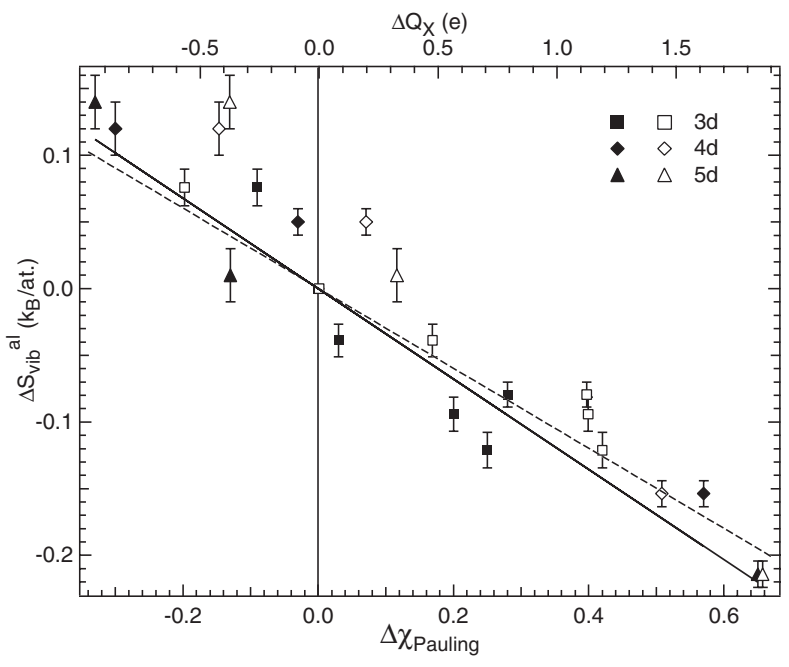

FIG. 4. Neutron-weighted vibrational entropy of alloying, $\Delta S_{\text {vib }}^{\mathrm{al}}$, plotted versus difference in Pauling electronegativity $\Delta \chi$ (solid symbols) between solute and $\mathrm{V}$, and Bader charge transfer on solute $\Delta Q_{\mathrm{X}}$ (open symbols). The solid (dashed) line is a linear fit of $\Delta S_{\mathrm{vib}}^{\mathrm{al}}$ versus $\Delta \chi\left(\Delta Q_{\mathrm{X}}\right)$.

atoms alters the phonons and the vibrational entropy. In a simple electrostatic model, the stiffness of the bonds is expected to increase with charge transfer and decrease with separation. The electrostatics of charge transfer and relaxation around the solutes are too large to explain the trends observed in the phonons, and a balance with other components of the electronic energy need be considered.

More fundamentally, it is the screening of ion motions by the electron density that governs the restoring forces on the ions and hence the phonon frequencies [21]. Our calculations show that the $d$ states of late transition metal solutes $\mathrm{Ni}, \mathrm{Pd}$, and Pt are lower in energy than the rest of the alloy valence bands, leading to a split band for Pd and Pt solutes and considerable distortions of the band shape. Correspondingly, we find a systematic depletion of the number of states at the Fermi level for solutes toward the end of the TM series (about 20\% for the Ni column). This is expected to cause a reduction in the number of weakly localized states available for screening the displacements of vanadium ions, causing higher energies for these displacements and higher phonon frequencies. From a chemistry viewpoint, larger differences in electronegativity will cause larger splittings between the bonding and antibonding states, making it harder to mix the states under a perturbation such as a phonon.

Because the effect of electronegative solutes on phonon stiffness originates with local bonds between neighboring solute and solvent atoms, this stiffening should continue with increasing solute concentration. Ordered structures tend to maximize the numbers of solute-solvent first neighbors, suggesting that similar investigations on orderdisorder transitions may be fruitful. As a start, we reassessed results on the vibrational entropy of formation for the $L 1_{2}$ alloys reported in [5]. We find that when an $L 1_{2}$ compound is formed with a minority species of a larger electronegativity than the majority species, the phonon DOS stiffens and the vibrational entropy decreases. The trend is approximately linear with electronegativity difference, although the potency of each solute atom is lower for $L 1_{2}$ alloys than for the $\mathrm{V}$ alloys studied here. There is no reason to expect that the observed behavior should be limited to vanadium alloys or to alloys of low solute concentration.

We thank Haibin Su for interesting discussions. This work was supported by the Department of Energy through Basic Energy Sciences Grant No. DE-FG02-03ER46055 and BES-MS, W-31-109-ENG-38.

[1] L. Anthony, J. K. Okamoto, and B. Fultz, Phys. Rev. Lett. 70, 1128 (1993).

[2] B. Fultz, L. Anthony, L. J. Nagel, R. M. Nicklow, and S. Spooner, Phys. Rev. B 52, 3315 (1995).

[3] V. Ozolins, C. Wolverton, and A. Zunger, Phys. Rev. B 57, 6427 (1998); 58, R5897 (1998).

[4] O. Delaire, T. Swan-Wood, and B. Fultz, Phys. Rev. Lett. 93, 185704 (2004).

[5] P. D. Bogdanoff and B. Fultz, Philos. Mag. B 81, 299 (2001).

[6] A. van de Walle and G. Ceder, Rev. Mod. Phys. 74, 11 (2002).

[7] See EPAPS Document No. E-PRLTAO-97-064650 for supplementary results on charge redistribution and phonon entropy of vanadium alloys. This EPAPS document describes the detailed numerical values for the measured and calculated properties of a series of $\mathrm{V}-6.25 \% \mathrm{X}$ bcc solid solutions, with $\mathrm{X}$ a transition metal solute. For more information on EPAPS, see http://www.aip.org/pubservs/ epaps.html.

[8] P. D. Bogdanoff, B. Fultz, and S. Rosenkranz, Phys. Rev. B 60, 3976 (1999).

[9] G. Placzek and L. Van Hove, Phys. Rev. 93, 1207 (1954).

[10] G. F. Syrykh et al., Sov. Phys. JETP 43, 183 (1976).

[11] P. Blaha, K. Schwarz, and P. Sorantin, Comput. Phys. Commun. 59, 399 (1990).

[12] G. Kresse and J. Furthmüller, Comput. Mater. Sci. 6, 15 (1996).

[13] G. Kresse and J. Joubert, Phys. Rev. B 59, 1758 (1999).

[14] J. P. Perdew, K. Burke, and M. Ernzerhof, Phys. Rev. Lett. 77, 3865 (1996).

[15] J. P. Perdew and Y. Wang, Phys. Rev. B 45, 13244 (1992).

[16] H. J. Monckhorst and J. D. Pack, Phys. Rev. B 13, 5188 (1976).

[17] R. Bader, Atoms in Molecules: A Quantum Theory (Oxford University Press, New York, 1990).

[18] G. Henkelman, A. Arnaldsson, and H. Jónsson, Comput. Mater. Sci. 36, 354 (2006).

[19] F. de Proft et al., J. Comput. Chem. 23, 1198 (2002).

[20] R.E. Watson and L. H. Bennett, Phys. Rev. B 18, 6439 (1978).

[21] K.-M. Ho, C.-L. Fu, and B. N. Harmon, Phys. Rev. B 29, 1575 (1984). 